\title{
Composición y estructura de la transición bosque-páramo en el corredor Guantiva-La Rusia (Colombia)
}

\author{
Jeison Adrián Olaya-Angarita ${ }^{1}$, Carlos Nelson Díaz-Pérez ${ }^{1}$ \& María Eugenia Morales-Puentes ${ }^{1}$ \\ 1. Sistemática Biológica, Herbario UPTC, Escuela de Biología \& Doctorado en Ciencias Biológicas y Ambientales, \\ Universidad Pedagógica y Tecnológica de Colombia, Avenida Central del Norte 39-115, 150003 Tunja, Boyacá, \\ Colombia; jeisonbiologiauptc@gmail.com,cndiazperez@gmail.com,maria.morales@uptc.edu.co
}

Recibido 22-II-2019. C Corregido 24-VI-2019. Aceptado 26-VI-2019.

\begin{abstract}
Composition and structure of the forest-páramo transition in the Guantiva-La Rusia corridor (Colombia). The altitudinal forest - paramo transition area, in its lower limit is characterized by the transition through the high Andean forest to high and low forest bushes, and is upper limit to the turnover between the low shrubs to herbaceous. Samplings were carried out in three localities of the Guantiva - La Rusia complex in the Western flank, through a $100 \mathrm{~m}^{2}$ parcels for the forest coverage and $50 \mathrm{~m}^{2}$ for the shrub and the herbaceous coverages, with an altitudinal difference of $100 \mathrm{~m}$ between altitudinal areas. We recorded 70 families, distributed in 157 genera and 302 species. The Asteraceae family were the most diverse with 16 genera and 39 species; likewise, Miconia was the most diverse with 14 species. Diversity indexes revealed that the most dominant in species composition were the herbaceous areas; regarding to diversity, the Onzaga-Susacon forest is 1.9 and 1.3 times more diverse than Belen and Duitama respectively. This research allowed identifying the transitional area through the transformation of the vascular flora physiognomy and diversity in the altitudinal gradient Western flank complex, that transition area is in Duitama between 3394 - $3516 \mathrm{~m}$, in Belen 3411 - $3649 \mathrm{~m}$ and in Onzaga-Susacon 3300 - 3390 m.
\end{abstract}

Key words: tropical treeline; structure; vegetation; floristic composition; paramo.

Olaya-Angarita, J. A., Díaz-Pérez, C. N., \& Morales-Puentes, M. E. (2019). Composición y estructura de la transición bosque-páramo en el corredor Guantiva-La Rusia (Colombia). Revista de Biología Tropical, 67(4), 755-768.

Los eventos geológicos y climáticos que han ocurrido a través del tiempo, han provocado que en los Andes tropicales exista una gran cantidad de hábitats terrestres. Estos ocasionan que la composición y estructura de la vegetación sean altitudinalmente variables, favoreciendo la alta heterogeneidad y diversidad de especies, que con el tiempo se han tornado propias en cada lugar (Churchill, Balslev, Forero, \& Luteyn, 1995; Webster, 1995; van der Hammen, 2000; van der Hammen \& Hooghiemstra, 2001; van der Hammen \& Otero-García, 2007).
Colombia por su posición geográfica ecuatorial en Sudamérica, es uno de los países con la mayor diversidad biótica del mundo, posee tres cordilleras, que alojan una gran cantidad de áreas ocupadas por ecosistemas naturales que mantienen una enorme riqueza florística (Bernal, Gradstein, \& Celis, 2016). Sin embargo, las acciones antrópicas como la expansión de la frontera agrícola, ganadera, quemas y erosión de suelos, han devastado estas áreas (Gentry, 1991; 1995; van der Hammen \& Rangel-Ch., 1997; Serrano, 2007). 
Los páramos son ecosistemas que se reconocen por su alta diversidad (Smith \& Cleef, 1988; Sklenár, Hedberg \& Cleef, 2014), comparados con otros ecosistemas similares en el mundo. Son propios del Neotrópico y se ubican en la parte alta de las montañas entre la franja de bosque andino y la parte más baja de las nieves (Rangel-Ch, 2000; Hofstede, Segarra, \& Mena, 2003; Llambí \& Cuesta, 2014). Ocupan el $2.5 \%$ del territorio colombiano (Sarmiento, Cadena, Sarmiento, Zapata, \& León, 2013) y brindan servicios ecosistémicos, como la regulación y constante provisión de agua (RangelCh., 2000), albergan un alto número de especies nativas y endémicas (Hofstede et al., 2003).

Actualmente en Colombia la delimitación de la franja de transición o ecotono del bosque y páramo, ha dado origen a una extensa discusión, debido a que se han instaurado leyes (ley 99/1993) que permiten la protección y conservación de los páramos, pero no se tiene una noción clara de la delimitación de dicha franja (Llambí, 2015). La franja bosque-páramo es definida por Bader, Rietkerk, \& Bregt (2007a), como una zona de transición, donde confluyen especies del bosque y la vegetación achaparrada de páramo; tal franja puede variar, debido a la alta heterogeneidad espacial de las cordilleras y los Andes en general (Bader van Geloof \& Rietkerk, 2007b; Ataroff \& GarcíaNúñez, 2013). Por otra parte, van der Hammen (1998), Ramírez, Llambí, Schwarzkopf, Gámez y Márquez (2009) y Arzac, Chacón-Moreno, Llambí, y Dulhoste (2011), sostienen que esta zona se caracteriza por tener una gran variabilidad en composición florística. A su vez, Harsch \& Bader (2011) definen para esta franja cuatro formas o patrones espaciales (difuso, abrupto, isla o lengüetas y "krummholz" en alemán madera doblada) basados en mecanismos de tres niveles, primero (limitación en el crecimiento, pérdida de biomasa y mortalidad de plántulas), segundo (estrés: fotoinhibición, daño por congelamiento, vientos, otros) y tercero (interacción con sus vecinos). Lo anterior, está relacionado con la dinámica de cada franja, debido a que integran factores abióticos (topografía, suelos, altitud, latitud, vientos, entre otros) y bióticos (composición de especies, respuestas fisiológicas, ente otros) (RangelCh., 2000; Vargas \& Pedraza, 2004; Sexton, McIntyre, Angert, \& Rice, 2009). Sin embargo, llegar a un acuerdo en la delimitación de la franja no ha sido posible. Este trabajo busca conocer desde el componente florístico (composición y estructura) una aproximación a la franja de transición entre el bosque y el páramo en el flanco occidental del complejo de páramos Guantiva-La Rusia en los departamentos de Boyacá y Santander.

\section{MATERIALES Y MÉTODOS}

Área de estudio: El complejo GuantivaLa Rusia (GL) es un corredor de páramos, bosque andino y alto andino, ubicado en el flanco occidental de la cordillera Oriental de los Andes colombianos. El estudio se realizó en los municipios de Duitama, Belén (páramo de La Rusia) y Onzaga-Susacón (páramo de Güina), en los departamentos de Boyacá y Santander. En Duitama (5'56'20.4" - 5'57'1.4" N \& 735>44.3" - 736'9.6” W), el páramo es el ecosistema más estudiado del complejo, y presenta alta diversidad florística (Morales et al., 2007; Martínez \& Díaz, 2017), posee una extensión de 10381 ha y presenta afectaciones humanas (Mora-Osejo \& Sturm, 1994); Belén (61'33.8” - 6²'50.4" N \& 72 $57^{\circ}$ '57" - 73'1'2.6” W), se encuentra al nor-oriente de Boyacá, con una extensión de páramo de 6080 ha; Onzaga-Susacón (6¹2'45.6" - 6¹3'9.4" $\mathrm{N} \& 72^{\circ} 46^{\prime} 42.2^{\prime \prime}-72^{\circ} 47^{\prime} 7.7^{\prime \prime} \mathrm{W}$ ) al sur de Santander y norte de Boyacá, con un área de páramo de 13033 ha (Fig. 1).

Muestreo: En cada localidad se realizó un transecto altitudinal con cuatro (Duitama, Onzaga-Susacon) y cinco (Belén) estaciones de muestreo cada $100 \mathrm{~m}$ (desde el bosque hasta el herbazal con presencia de frailejones). En cada estación se ubicaron tres parcelas; para evaluar el bosque fueron instaladas parcelas de $4 \times 25$ $\mathrm{m}\left[100 \mathrm{~m}^{2}\right.$, divididas en subparcelas de $4 \times 5$ $\mathrm{m}\left(20 \mathrm{~m}^{2}\right)$ ], para arbustal y herbazal parcelas de $4 \times 12.5 \mathrm{~m}\left[50 \mathrm{~m}^{2}\right.$, divididas en subparcelas 


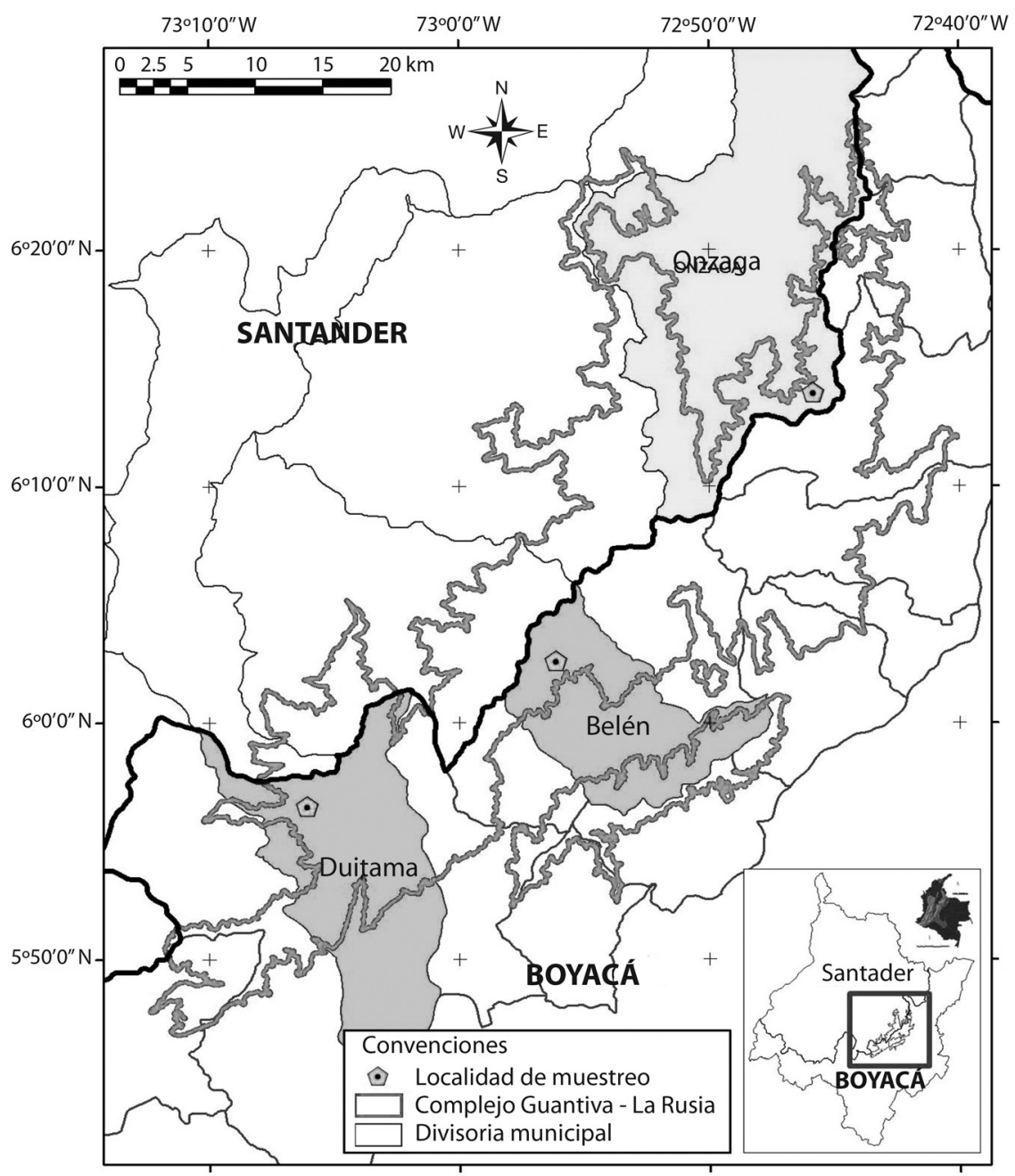

Fig. 1. Área de estudio, delimitación del complejo de páramos Guantiva-La Rusia.

Fig 1. Study area, delimitation of the paramo Guantiva-La Rusia complex.

de 4 x $2.5 \mathrm{~m}\left(10 \mathrm{~m}^{2}\right)$ ] (Marín, 2013). Se evaluaron las siguientes variables: altura $(\mathrm{m})$, diámetro a la altura del pecho (DAP), cobertura (Rangel-Ch. \& Velásquez, 1997), abundancia y riqueza. Para las herbáceas se midió por datos porcentuales y de frecuencia en cada parcela. El material fue depositado en los Herbarios UPTC y FMB (Thiers, 2018), bajo la clasificación APG IV (2016).

Análisis de datos: Se determinó la riqueza y composición florística con base en el número de familias, géneros y especies, en cada uno de los sitios de muestreo. Seguidamente en cada estación de muestreo, se determinaron las formaciones vegetales, a partir de la cobertura relativa de los hábitos de crecimiento: árbol, arbolito, arbustillo, arbusto, hierba, roseta, macolla, entre otros (Marín, 2013). Se calculó el índice de diversidad alfa según Moreno, Barragán, Pineda, y Pavón (2011), que mide la diversidad de una comunidad integrada por $i$ especies con igual número de individuos $\left[\mathrm{p}_{1}\right.$ $=\mathrm{p}_{2}=\mathrm{p}_{3}=\mathrm{p}_{\mathrm{s}}$ ], mediante el número efectivo de especies a nivel de formaciones vegetales dentro de cada localidad, con el uso de la 
diversidad de orden 1 (q1). Para establecer las diferencias en la composición de la vegetación, dentro y entre las localidades se organizó la información teniendo en cuenta la presenciaausencia de especies por estación, y se usó para llevar a cabo el análisis de diversidad beta, a través del paquete R Betapart (Baselga, 2012), con las funciones "beta-multi" la cual permite obtener el recambio ( $\beta$ sim - disimilaridad de Simpson) y anidamiento ( $\beta$ sne - resultante de la fracción de disimilaridad de Sorensen). Además, se empleó el "beta-pairwise" que incluye las funciones para calcular matrices de distancia, con el uso de diferencias por pares ( $\beta$ sor) para hacer un análisis de conglomerados. Adicionalmente, se calculó el índice de Whittaker (1972) con el fin de conocer las comunidades o agrupamientos entre estaciones.

\section{RESULTADOS}

Riqueza: Se registraron 70 familias, 157 géneros y 302 especies (Apéndice 1). La familia Asteraceae es la más diversa con 16 géneros y 39 especies, seguida de Ericaceae (11/19), Poaceae (11/12), Orchidaceae (10/14) y Melastomataceae (7/20). El género más diverso es Miconia con 14 especies, seguido de Elaphoglossum (9), Hypericum (7), Ageratina y Pentacalia con seis cada una. La riqueza de familias y géneros por localidad no presentó mayores diferencias. La localidad con mayor número de especies fue Belén con 176, que representan el $58 \%$ del total (Tabla 1 ).

Formación vegetal: Se determinó la estructura y tipo de formación vegetal, así: en

TABLA 1

Coberturas vegetales, riqueza florística, rango altitudinal por estación y diversidad verdadera alfa por cobertura en cada localidad, Guantiva-La Rusia

TABLE 1

Plant coverings, floristic richness, altitudinal range by season and true alpha diversity by coverage in each location, Guantiva-La Russia complex

\begin{tabular}{|c|c|c|c|c|c|c|}
\hline Localidad & $\begin{array}{c}\text { Estación } \\
\text { (cobertura vegetal) }\end{array}$ & $\begin{array}{c}\text { Rango } \\
\text { altitudinal (m) }\end{array}$ & $\begin{array}{c}\text { No. } \\
\text { Familias }\end{array}$ & $\begin{array}{c}\text { No. } \\
\text { Géneros }\end{array}$ & $\begin{array}{c}\text { No. } \\
\text { Especies }\end{array}$ & $\begin{array}{l}\text { Diversidad verdadera alfa } \\
\text { por cobertura vegetal } \\
\text { (q1) }\end{array}$ \\
\hline \multirow{5}{*}{ 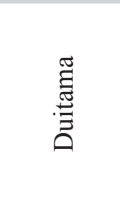 } & DE1 (Bosque) & $3265-3286$ & 34 & 48 & 58 & 34.01 \\
\hline & DE2 (Arbustal) & $3394-3434$ & 34 & 51 & 61 & \multirow{2}{*}{29.09} \\
\hline & DE3 (Arbustal) & $3490-3516$ & 23 & 44 & 48 & \\
\hline & DE4 (Herbazal) & $3593-3615$ & 20 & 31 & 38 & 13.57 \\
\hline & Total & - & 56 & 105 & 147 & 44.17 \\
\hline \multirow{6}{*}{$\frac{\sqrt{0}}{\stackrel{0}{0}}$} & BE1 (Bosque) & $3275-3336$ & 29 & 38 & 48 & 23.56 \\
\hline & BE2 (Arbustal) & $3411-3430$ & 19 & 31 & 35 & \multirow{3}{*}{41.21} \\
\hline & BE3 (Arbustal) & $3499-3505$ & 28 & 48 & 59 & \\
\hline & BE4 (Arbustal) & $3642-3649$ & 34 & 56 & 68 & \\
\hline & BE5 (Herbazal) & $3766-3776$ & 19 & 30 & 39 & 12.63 \\
\hline & Total & - & 54 & 108 & 176 & 66.92 \\
\hline \multirow{6}{*}{ 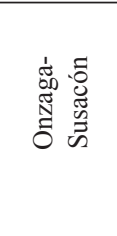 } & OE1 (Bosque) & $2944-3129$ & 35 & 48 & 55 & \multirow{2}{*}{45.49} \\
\hline & OE2 (Bosque) & $3191-3200$ & 37 & 50 & 60 & \\
\hline & OE3 (Arbustal) & $3300-3313$ & 27 & 46 & 49 & 25.72 \\
\hline & OE4 (Herbazal) & $3444-3456$ & 14 & 25 & 27 & 11.66 \\
\hline & Total & - & 55 & 102 & 141 & 68.00 \\
\hline & \multicolumn{2}{|c|}{ Total, en las tres localidades } & 70 & 157 & 302 & \\
\hline
\end{tabular}


Duitama, la estación uno (E1) se definió como bosque, por el predominio de árboles con 88.71 $\%$, seguido de arbolitos (5.24 \%). La estación dos (E2) es un arbustal con el $70.20 \%$ de arbustos, seguido de arbustillos (16.05\%). La estación tres (E3) es un arbustal, con predominio de arbustillos y arbustos (46.03 y $42.82 \%$ respectivamente). La estación cuatro (E4) es un herbazal con predominio de macollas (32.30 $\%$ ) seguidas de arbustillos (23.46\%).

En Belén los hábitos dominantes en la estación uno (E1), los árboles con el 90.83\%, seguida de arbustos con $3.44 \%$, y se definió como bosque. La estación dos (E2) es un arbustal con predominio de arbustillos y arbustos con el 38.06 y $37 \%$ respectivamente. La estación tres (E3) es un arbustal con arbustos $(78.85 \%)$ y arbustillos $(6.44 \%)$. La estación cuatro (E4) es un arbustal con arbustos (42.24 $\%)$ y arbolitos (26.80\%), y la estación cinco (E5) es un herbazal con macollas (71.69\%) seguida de, rosetas con un solo tronco $(9.78 \%)$.

En Onzaga-Susacón, las estaciones uno y dos corresponden a bosque por sus hábitos arbóreos (E1-71.61, E2-74.05 \%) y arbustivos (E1-14.67, E2-13.56 \%). La estación tres (E3) es un arbustal con arbustos y arbustillos (67.91 y $9.91 \%$ respectivamente), y la estación cuatro (E4) es un herbazal donde las macollas representan el $71.56 \%$, seguidas del hábito chusqueoide $(7.11 \%)$ (Tabla 1$)$.

Según las formas propuestas por Harsch y Bader (2011), se registran para Duitama y Onzaga-Susacón, una franja de transición con forma difusa y para Belén, en islas o lengüetas. En los herbazales se destacan especies de los géneros Achyrocline, Baccharis, Espeletia, Chusquea, Hypericum, Hypochaeris, Oreobolus y Halenia, en los arbustales, Vaccinium, Gaultheria, Hypericum, Ageratina, Berberis, Bejaria, Dendrophthora, Miconia y Peperomia y en los bosques Brunellia, Clusia, Anthurium, Miconia, Quercus, Solanum, Clethra y Weinmannia; adicionalmente, se registraron 35 especies propias de los herbazales que corresponden al $42.6 \%$ del total registrado, 93 (54.7 \%) para los arbustales y 96 (73.8\%) para los bosques, junto con 41 especies compartidas entre las formaciones vegetales de bosque y arbustal, entre arbustal y herbazal se comparten 30 especies y para las tres formaciones vegetales se hallaron seis especies compartidas.

Diversidad: la mayor diversidad se registró para el transecto de Onzaga-Susacón con 68 especies efectivas, seguido de Belén (66.92) y Duitama (44.17) (Tabla 1). El bosque de Onzaga-Susacón registra 1.9 y 1.3 veces, más diversidad que Belén y Duitama respectivamente. Sin embargo, esta localidad registra el valor más bajo para el arbustal con 25.72 especies efectivas. El herbazal más diverso corresponde a Duitama con 1.07 y 1.16 veces más diverso que Belén y Onzaga-Susacón.

Diversidad beta: este análisis mostro que el recambio espacial de especies $(\beta \operatorname{sim}=$ 0.85 ) es alto entre las estaciones evaluadas; mientras que el anidamiento fue bajo ( $\beta$ sne $=$ 0.09). Se registran cinco agrupamientos que corresponden: a) estaciones de bosque, que se subdividen en dos comunidades (BE1 - DE1 y OE1 - OE2); b) estaciones de herbazales (BE5 y DE4) con una disimilitud del $67 \%$; c) la estación arbustal de Onzaga-Susacón (OE3) con el $70 \%$ de disimilitud; d) una estación de arbustal de Belén (BE2) y una estación de herbazal de Onzaga-Susacón (OE4) y e) estaciones de arbustal de Duitama y Belén (BE4, DE2, BE3 y DE3. El índice de Whittaker registra cinco comunidades (5.0667), las cuales coinciden con las mencionadas anteriormente (Fig. 2).

\section{DISCUSIÓN}

Las familias con mayor riqueza en géneros y especies aquí registradas, son similares a otros estudios realizados en los ecosistemas de alta montaña (Rangel-Ch., 2000; Briceño \& Morillo, 2006; Lozano, Cleef, \& Bussmann, 2009; Cuello, Cleef, \& Aymard, 2010). A lo largo del flanco occidental se encontró un alto grado de semejanzas en la composición de familias (77 - $80 \%$ ), géneros (64 - $69 \%)$, y menor proporción de especies (46 - $58 \%$ ). 


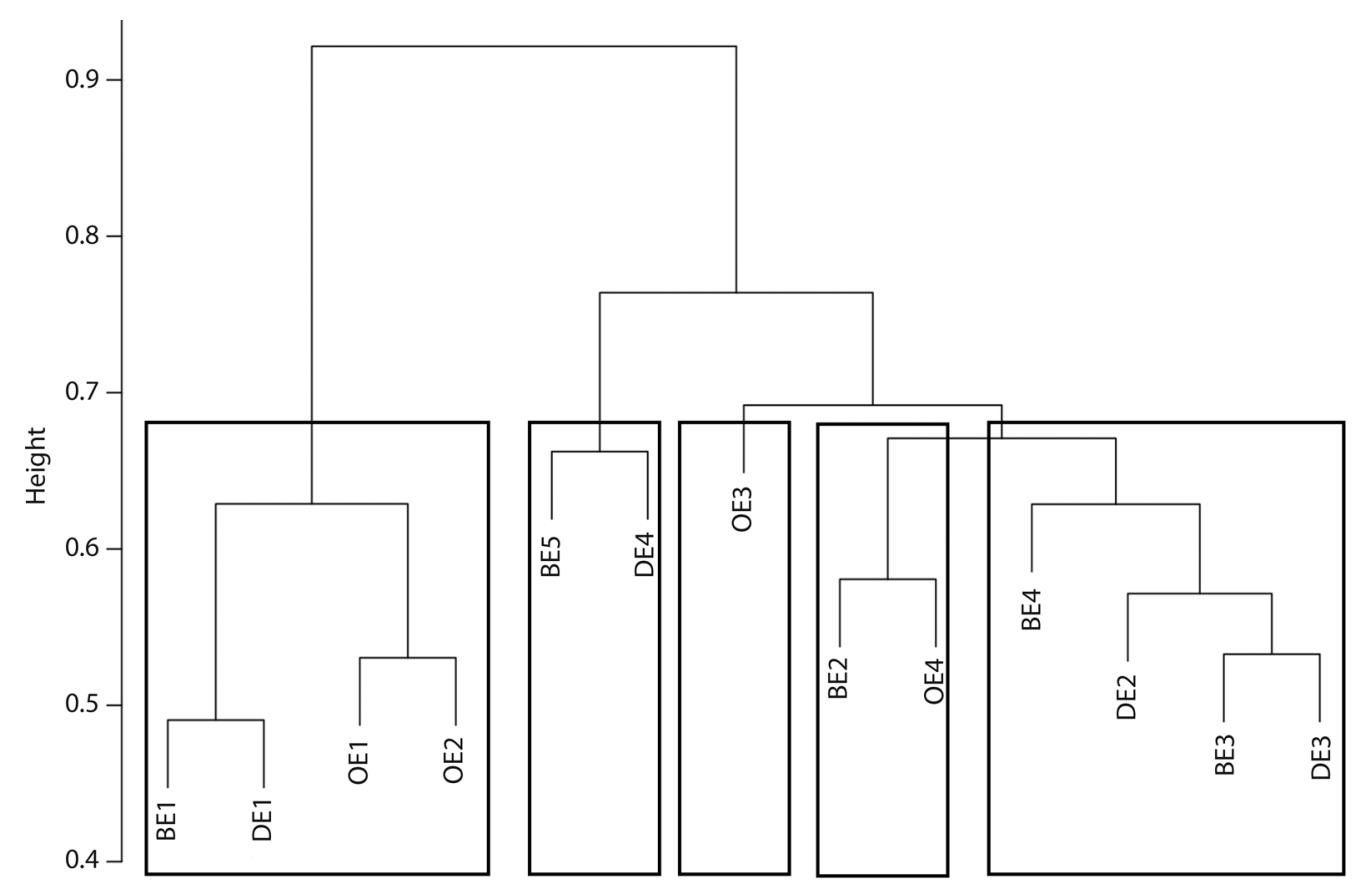

Fig. 2. Agrupamiento a partir del análisis de diversidad beta entre las estaciones (E) de Duitama (D), Belén (B) y OnzagaSusacón (O), en el costado occidental del complejo de páramos Guantiva-La Rusia (Boyacá-Santander).

Fig. 2. Grouping based on the analysis of beta diversity between the stations (E) of Duitama (D), Belén (B) and OnzagaSusacón $(\mathrm{O})$, on the western side of the páramos Guantiva-La Rusia complex (Boyacá-Santander).

En las localidades de Duitama y OnzagaSusacón, la mayor diversidad de especies se encontró en las estaciones de bosque, lo que concuerda con estudios realizados por Cuatrecasas (1958), Gentry (1995), Galindo, Betancur, y Cadena (2003), quienes afirman que la diversidad vegetal es mayor en los transectos de altitudes bajas y es menor en las zonas altas. Pese a esto, los bosques entre sí, presentaron diferencias notables, lo cual indica que, aunque son formaciones vegetales estructuralmente similares poseen riqueza variable entre ellos (Galindo et al., 2003).

La alta diversidad del arbustal en Belén, se explica por los efectos de transformación de las formaciones vegetales originales, como consecuencia de la tala, quema, ganadería y cultivos (papa), que históricamente se han visto en la zona e igualmente han sido registrados por otros autores en los Andes (Serrano, 2007; Velasco-Linares \& Vargas, 2008; Sarmiento \&
Llambí, 2011). Tales eventos, junto con la inclinación del terreno han favorecido la llegada de especies arbustivas que soportan las nuevas condiciones de las áreas transformadas. Esta modificación ha ocasionado en esta localidad que dicha franja tenga un rango altitudinal (300 m) mayor a las demás, lo que se ve reflejado en un mayor muestreo (tres estaciones) y pueda ser la causa de presentar una mayor diversidad. Lo anterior, se argumenta con la teoría del disturbio intermedio de Connell (1978), que indica, que una alta diversidad de especies es generada luego de un disturbio antrópico.

Las estaciones con dominancia de estratos arbóreos fueron: uno de Duitama (3265 - 3286 m), Uno de Belén (3275 - 3336 m), y uno - dos de Onzaga-Susacón (2944 $3200 \mathrm{~m}$ ) que corresponde a bosque altoandino, observaciones similares fueron realizadas por Acosta-Solis (1984), Bader et al., (2007a), van der Hammen (1998), Ramírez et al. (2009) y 
Arzac et al. (2011), quienes indican que estos bosques en su límite superior están dominados por árboles y arbustos. Por otro lado, RangelCh. (2000) en sus estudios menciona que los géneros Weinmannia y Clethra son dominantes en los bosques andinos, lo cual coincide con lo encontrado en este trabajo (Weinmannia tomentosa, W. fagaroides y Clethra fimbriata), junto con Quercus humboldtii los más dominantes.

La amplitud de la franja de transición bosque-páramo en el flanco, no es uniforme; se encontró que oscila entre 3300 y 3649 m, contrastado con lo mencionado por autores como, Ataroff \& Sarmiento (2004), Rivera \& Rodríguez (2011) y Ataroff \& García-Núñez (2013), quienes definen dicha franja entre el rango de los 2700 y $3000 \mathrm{~m}$; lo anterior, se debe a factores de tipo biótico y abiótico que afectan la amplitud de los límites de cada localidad.

Monasterio (1980) señala que en la franja de transición bosque-páramo se distribuyen especies arbóreas que conforman islas de bosques, las cuales según Llambí (2015) están dominadas por familias como Myrsinaceae, Rosaceae, Clusiaceae y Asteraceae; no obstante, en el presente estudio se encontraron las mismas familias, integrando estas islas, y se hallaron también, Cunoniaceae, Melastomataceae y Symplocaceae. A su vez, se registró en estos fragmentos una diversidad menor a la línea continua del bosque, como fue afirmado por Jørgensen y Ulloa-Ulloa (1994), Wille, Hooghiemstra, Hofstede, Fehse, y Sevink (2002) y Ramírez et al. (2009), donde plantean que estas formaciones son distintas al bosque y están adaptadas a las condiciones del ecosistema donde se encuentran.

Cuatrecasas (1958), Luteyn (1999) y Rangel-Ch. (2000) afirman que el páramo propiamente dicho, está dominado por macollas, rosetas, hierbas y arbustos enanos. Sin embargo, en este trabajo se encontraron algunas especies dispersas, como arbolitos y arbustos, característicos del bosque andino; así también, se registraron especies de Hypericum, Espeletia, Espeletiopsis, Arcytophyllum, Calamagrostis y Chusquea como dominantes en los herbazales de las localidades muestreadas. Otro aspecto relevante, corresponde a la variación registrada en el límite inferior en cada localidad del herbazal así, para Duitama se encuentra en la cota de los $3593 \mathrm{~m}$, en Belén desde los 3766 m y Onzaga-Susacón a 3444 m. Sin embargo, Rangel-Ch. (2000) mencionó que el páramo se encuentra sobre los $3500 \mathrm{~m}$, lo cual difiere con lo registrado en este trabajo.

La diversidad verdadera reflejó que el páramo de Onzaga-Susacón fue el más diverso (68) con relación a las demás áreas de estudio; a su vez, la diversidad es mayor hacia el oriente del complejo en una proporción de 1.5 veces más diverso Onzaga-Susacón que Duitama. Esto puede estar favorecido por presentar un mayor régimen de humedad, debido a la ubicación geográfica y las lluvias orogénicas provenientes del valle del Magdalena (Morales et al., 2007).

A partir del análisis de diversidad beta, se observa que las estaciones de bosque, y los herbazales de Duitama y Belén, pueden ser definidas tanto por composición de especies como por su estructura; sin embargo, el herbazal de Onzaga-Susacón se encuentra más relacionado con las estaciones de arbustal, excepto la de su misma localidad, con la cual, se registra un $70 \%$ de disimilitud. Lo anterior, muestra que, para el área de estudio la composición de especies se encuentra relacionada con la altitud, debido a que el transecto de Onzaga-Susacón se encuentra por debajo de las otras localidades.

A partir de los agrupamientos a nivel de familias, se encontró que la vegetación de arbustales, comparte una mayor similitud con la de herbazales, lo cual indica que hay especies del páramo que han migrado hacia la zona de transición bosque-páramo, ello sugiere que se considere como área de conservación junto con los páramos, ya que juegan un papel fundamental en la preservación de especies de la alta montaña.

La dinámica de la franja de transición entre el bosque y el páramo en la cordillera Oriental, juega un rol importante a la hora de conocer procesos bióticos que allí ocurren; conocer la diversidad de estas áreas es fundamental para 
que sean tenidas en cuenta para la toma de decisiones a nivel nacional, por ende, estudios de este tipo son necesarios para la protección y preservación de dichas áreas.

Declaración de ética: los autores declaran que todos están de acuerdo con esta publicación y que han hecho aportes que justifican su autoría; que no hay conflicto de interés de ningún tipo; y que han cumplido con todos los requisitos y procedimientos éticos y legales pertinentes. Todas las fuentes de financiamiento se detallan plena y claramente en la sección de agradecimientos. El respectivo documento legal firmado se encuentra en los archivos de la revista.

\section{AGRADECIMIENTOS}

A la Universidad Pedagógica y Tecnológica de Colombia, y al Convenio No. 14-13-014-195CE entre UPTC-IAvH con el proyecto "caracterización biótica como soporte en la delimitación de páramos de los complejos Guantiva-La Rusia y Mamapacha-BijagualTota en los departamentos de Boyacá y Santander". Al grupo de investigación Sistemática Biológica (SisBio) y al Herbario UPTC. A las comunidades locales de Duitama, Belén, Onzaga y Susacón; Jorge Mercado y Alexander Sabogal; así como a cada uno de los que de una $\mathrm{u}$ otra forma hicieron parte de este proyecto.

\section{RESUMEN}

La franja de transición altitudinal bosque-páramo, en su límite inferior se caracteriza por la transición de bosque altoandino a arbustales de portes alto y bajo, y en su límite superior de arbustales de bajo porte a herbazales. En tres localidades del flanco occidental del complejo de páramos Guantiva - La Rusia se realizaron muestreos, en parcelas de $100 \mathrm{~m}^{2}$ para la cobertura de bosque y $50 \mathrm{~m}^{2}$ para la arbustal y herbazal, con una diferencia altitudinal de 100 $\mathrm{m}$ aproximadamente entre las estaciones. Se encontraron 70 familias, distribuidas en 157 géneros y 302 especies, siendo Asteraceae la familia más diversa con 16 géneros y 39 especies, a su vez, Miconia el género más diverso con 14 especies. Los índices de diversidad revelaron que las estaciones de herbazales, para las tres localidades fueron las más dominantes en composición de especies; en cuanto a la diversidad el bosque de Onzaga-Susacón registra 1.9 y 1.3 veces, más diversidad que Belén y Duitama, respectivamente. El presente estudio permitió identificar la franja de transición a partir del cambio en la fisionomía y diversidad de la flora vascular en el gradiente altitudinal del flanco occidental del complejo, dicha franja se ubica en Duitama entre los 3394 y 3516 m de altitud, en Belén entre los 3411 y 3649 m y en Onzaga-Susacón entre los 3300 y 3390 m.

Palabras clave: límite de bosque; estructura; vegetación; composición florística; páramo.

\section{REFERENCIAS}

Acosta-Solis, M. (1984). Los páramos andinos del Ecuador. Quito. Ecuador: Publicaciones Científicas MAS.

Arzac, A., Chacón-Moreno. E., Llambí, L. D., \& Dulhoste, R. (2011). Distribución de formas de vida de plantas en el límite superior del ecotono bosque páramo en los Andes Tropicales. Ecotrópicos, 24(1), 26-46.

Ataroff, M., \& Sarmiento, L. (2004). Las unidades ecológicas de los Andes de Venezuela. En E. La Marca \& P. Soriano (Eds.), Reptiles de Los Andes de Venezuela (pp. 9-26). Mérida, Venezuela: Fundación Polar, Codepre-ULA, Fundacite-Mérida, Biogeos.

Ataroff, M., \& García-Núñez, C. (2013). Selvas y bosques nublados de Venezuela. En E. Medina, O. Huber, J. Nassar, \& P. Navarro. (Eds.), Recorriendo el paisaje vegetal en Venezuela (pp. 125-155). Caracas, Venezuela: Ediciones IVIC, Instituto Venezolano de Investigaciones Científicas.

Bader, M., Rietkerk, M., \& Bregt, A. (2007a). Vegetation structure and temperature regimes of tropical alpine treelines. Arctic, Antarctic, and Alpine Research, 39(3), 353-364. DOI: 10.1657/1523-0430 (06-055) [BADER]2.0.CO;2

Bader, M., van Geloof, I., \& Rietkerk, M. (2007b). High solar radiation hinders tree regeneration above the alpine treeline in northern Ecuador. Plant Ecology, 191(1), 33-45. DOI: 10.1007/s11258-006-9212-6

Baselga, A. (2012). The relationship between species replacement, dissimilarity derived from nestedness, and nestedness. Global Ecology and Biogeography, $21,1223-1232$.

Bernal, R., Gradstein, S. R., \& Celis, M. (Eds.) (2016). Catálogo de plantas y líquenes de Colombia. Bogotá, Colombia: Universidad Nacional de Colombia.

Briceño, B., \& Morillo, G. (2006). Catálogo de las plantas con flores de los páramos de Venezuela. Parte II. Monocotiledóneas (Liliopsida). Acta Botánica Venezuelica, 29(1), 89-134. 
Churchill, S., Balslev, P., Forero, H., \& Luteyn, J. (1995). Biodiversity and conservation of Neotropical montane forest. The New York Botanical Garden, Nueva York, 103-126.

Connell, J. H. (1978). Diversity in tropical rain forests and Coral reefs. Science, New Series, 199(4335), $1302-1310$.

Cuatrecasas, J. M. (1958). Aspectos de la vegetación natural de Colombia. Academia Colombiana de Ciencias Exactas Físicas y Naturales, 10, 221-269.

Cuello, N., Cleef, A., \& Aymard, G. (2010). Phytogeography of the vascular paramo flora of Ramal de Guaramacal (Andes, Venezuela) and its ties to other paramo floras. Anales Jardín Botánico de Madrid, 67(2), 177-193.

Galindo, R., Betancur, J., \& Cadena, J. (2003). Estructura y composición florística de cuatro bosques andinos del santuario de flora y fauna Guanentá-Alto Río Fonce, cordillera Oriental colombiana. Caldasia, 25(2), 313-335.

Gentry, A. (1991). Vegetación del bosque de niebla. En C. Uribe (Ed.), Bosques de niebla de Colombia (pp. 23-52). Cali, Colombia: Banco de Occidente.

Gentry, A. (1995). Patterns of diversity and floristic composition in Neotropical montane forests. En S. P. Churchill, H. Balslev, E. Forero, \& J. L. Luteyn (Eds.), Biodiversity and conservation of Neotropical montane forests (pp. 103-133). New York, USA: The New York Botanical Garden.

Harsch, M. A., \& Bader, M. Y. (2011). Treeline form - a potential key to understanding treeline dynamics. Global Ecology and Biogeography, 20, 582-596.

Hofstede, R., Segarra, P., \& Mena, P. (Eds.). (2003). Los páramos del mundo. Proyecto atlas mundial de los páramos. Quito, Ecuador: Global Peatland Initiative/ NC-IUCN/EcoCiencia.

Jørgensen, P., \& Ulloa-Ulloa, C. (1994). Seed plants of the high Andes of Ecuador: a checklist. Aarhus, Denmark: Department of Systematic Botany, Aarhus University; AAU reports Department of Systematic Botany University of Aarhus.

Llambí, L. D., \& Cuesta, F. (2014). La diversidad de los páramos andinos en el espacio y en el tiempo. En F. J. Cuesta, L. Sevink, B. Llambí, \& J. Posner (Eds.), Avances en investigación para la conservación de los páramos andinos. Quito, Ecuador: CONDESAN.

Llambí, L. D. (2015). Estructura, diversidad y dinámica de la vegetación en el ecotono bosque-páramo: revisión de la evidencia en la cordillera de Mérida. Acta biológica Colombiana, 20(3), 5-20. DOI: 10.15446/-abc. v20n3.46721
Lozano, P., Cleef, A. M., \& Bussmann, R. (2009). Phytogeography of the vascular paramo flora of Podocarpus Biosphere Reserve, South Ecuador. Arnaldoa, 16(2), 69-85.

Luteyn, J. (1999). Paramos: A checklist of plant diversity, geographical distribution and botanical literature. New York Botanical Garden, 84, 1-278.

Marín, C. (2013). Propuesta metodológica para caracterizar las coberturas vegetales en los páramos de Santurbán y Rabanal. Proyecto páramos y sistemas de vida. Instituto de Investigación en Recursos Biológicos Alexander von Humboldt.

Martínez, M. F., \& Díaz, C. N. (2017). Riqueza de musgos por sustratos en un gradiente altitudinal del complejo de páramos Guantiva-La Rusia (Boyacá y Santander - Colombia). Ciencia en Desarrollo (Suplemento especial), 334-335.

Monasterio, M. (1980). Los páramos andinos como región natural. Características biogeográficas generales y afinidad con otras regiones andinas. En M. Monasterio (Ed.), Estudios ecológicos en los páramos andinos (pp. 15-27). Mérida: Editorial de la Universidad de Los Andes.

Mora-Osejo, F., \& Sturm, H. (1994). Estudios ecológicos del páramo y del bosque alto andino: cordillera Oriental de Colombia. Bogotá, Colombia: Academia Colombiana de Ciencias Exactas, Físicas y Naturales.

Morales, M., Otero, J., van der Hammen, T., Torres, A., Cadena, C., Pedraza, C., ... Cárdenas, L. (2007). Atlas de páramos de Colombia. Bogotá D.C., Colombia: Instituto de Investigación de Recursos Biológicos Alexander von Humboldt.

Moreno, C. E., Barragán, F., Pineda, E., \& Pavón, N. P. (2011). Reanálisis de la diversidad alfa: alternativas para interpretar y comparar información sobre comunidades ecológicas. Revista Mexicana de Biodiversidad, 82, 1249-1261.

Ramírez, L., Llambí, L. D., Schwarzkopf, T., Gámez, L. E., \& Márquez, N. J. (2009). Vegetation structure along the forest-paramo transition belt in the Sierra Nevada de Mérida: Implications for understanding treeline dynamics. Ecotropicos, 22(2), 83-98.

Rangel-Ch, J. O., \& Velázquez. A. (1997). Métodos de estudio de la vegetación. En O. Rangel, P. Lowy, \& P. M. Aguilar (Eds.), Colombia diversidad biótica II. Tipos de vegetación en Colombia (pp. 59-87). Bogotá, Colombia: Editorial Unibiblios.

Rangel-Ch., J. O. (2000). Colombia: diversidad biótica III, la región de vida paramuna. Bogotá, D. C., Colombia: Facultad de Ciencias, Universidad Nacional de Colombia - Instituto de Ciencias Naturales e Instituto Alexander von Humboldt. 
Rivera, D., \& Rodríguez, C. (2011). Guía divulgativa de criterios para la delimitación de páramos de Colombia. Bogotá, D. C., Colombia: Ministerio de Ambiente, Vivienda y Desarrollo Territorial e Instituto de Investigación de Recursos Biológicos Alexander von Humboldt.

Sarmiento, L., \& Llambí, L. D. (2011). Regeneración del páramo luego de un disturbio agrícola: una síntesis de 20 años de investigaciones en sistemas con descansos largos en la cordillera de Mérida. En F. Herrera, \& I. Herrera (Eds.), La restauración ecológica en Venezuela: fundamentos y experiencias (pp. 123-148). Caracas, Venezuela: Ediciones Instituto Venezolano de Investigaciones Científicas.

Sarmiento, C., Cadena, C., Sarmiento, M., Zapata, J., \& León, O. (2013). Aportes a la conservación estratégica de los páramos de Colombia: actualización de la cartografía de los complejos de páramo a escala 1:100.000. Bogotá, D.C., Colombia: Instituto de Investigación de Recursos Biológicos Alexander von Humboldt.

Serrano, C. (2007). Situación de los páramos en Colombia frente a la actividad antrópica y el cambio climático. Informe preventivo. Bogotá, Colombia: Instituto de Estudios del Ministerio Público. Procuraduría General de la Nación.

Sexton, J. P., McIntyre, P. J., Angert, A. L., \& Rice, K. J. (2009). Evolution and ecology of species range limits. Annual Reviews of Ecology, Evolution and Systematics, 40, 415-436.

Sklenár, P., Hedberg, I., \& Cleef, A. M. (2014): Island biogeography of tropical alpine floras. Journal of Biogeography, 14, 287-297. DOI: 1111/bji. 12212

Smith, J. M. B., \& Cleef, A. M. (1988). Composition and origins of the world's tropicalpine floras. Journal of Biogeography, 15, 631-645.

Thiers, B. (2018). Index Herbariorum: A global directory of public herbaria and associated staff. New York Botanical Garden's Virtual Herbarium. Retrieved from: http://sweetgum.nybg.org/science/ih

van der Hammen, T. (1998). Páramos. En M. E. Chaves, \& N. Arango (Eds.), Informe nacional sobre el estado de la biodiversidad 1997- Colombia. Bogotá, Colombia: Instituto de Investigación de Recursos Biológicos Alexander von Humboldt. PNUMA. Ministerio del Medio Ambiente. van der Hammen, T., \& Rangel, J. O. (1997). El estudio de la vegetación en Colombia. En J. O. Rangel-Ch, P. D. Lowy-C., \& M. Aguilar-P. (Eds.), Colombia Diversidad Biótica II, tipos de vegetación en Colombia (pp. 17-57). Bogotá, Colombia: Instituto de Ciencias Naturales, Universidad Nacional de Colombia.

van der Hammen, T. (2000). Aspectos de historia y ecología de la biodiversidad norandina y amazónica. Academia Colombiana de Ciencias Exactas, Físicas y Naturales, 24(91), 231-245.

van der Hammen, T., \& Hooghiemstra, H. (2001). Historia y paleoecología de los bosques montanos andinos neotropicales. En M. Kappelle, \& A. D. Brown (Eds.), Bosques nublados del Neotrópico (pp. 63-84). Santo Domingo de Heredia, Costa Rica: Instituto Nacional de Biodiversidad (INBio).

van der Hammen, T., \& Otero-García, J. (2007). Los páramos: archipiélagos terrestres en el norte de los Andes. En M. Morales, J. Otero, T. van der Hammen, A. Torres, C. Cadena, C. Pedraza, ... L. Cárdenas. Atlas de páramos de Colombia (pp. 25-31). Bogotá, Colombia: Instituto de Investigaciones de Recursos Biológicos Alexander von Humboldt.

Vargas, O., \& Pedraza, P. (2004). Parque Nacional Natural Chingaza. Bogotá, Colombia: Universidad Nacional, Colciencias, Parques Nacionales, Acueducto de Bogotá.

Velasco-Linares, P., \& Vargas, O. (2008). Problemática de los bosques alto andinos. En O. Vargas (Ed.), Estrategias para la restauración ecológica del bosque alto andino (El caso de la Reserva Forestal Municipal de Cogua, Cundinamarca) (pp. 41-56). Bogotá, Colombia: Universidad Nacional de Colombia.

Webster, G. L. (1995). The panorama of Neotropical cloud forests. En S. P. Churchill, H. Balslev, E. Forero, \& J. L. Luteyn (Eds.), Biodiversity and conservation of Neotropical montane forest (pp. 53-77). New York, USA: The New York Botanical Garden.

Whittaker, R. H. (1972). Evolution and measurement of species diversity. Taxon, 21(2/3), 213-251.

Wille, M., Hooghiemstra, H., Hofstede, R., Fehse, J., \& Sevink, J. (2002)er forest line reconstruction in a deforested area in northern Ecuador based on pollen and vegetation analysis. Journal Tropical Ecology, 18(3), 409-440. 


\section{APÉNDICE 1}

Especies por localidad, presencia (X)

\section{APPENDIX 1}

List of species by location, presence $(\mathrm{X})$

\begin{tabular}{|c|c|c|c|}
\hline Especie & 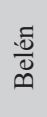 & 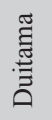 & 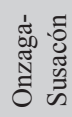 \\
\hline Acaena cylindristachya & $\mathrm{X}$ & & $\mathrm{X}$ \\
\hline Acaena elongata & $\mathrm{X}$ & & \\
\hline Achyrocline & & $\mathrm{X}$ & \\
\hline Achyrocline bogotensis & $\mathrm{X}$ & $\mathrm{X}$ & $\mathrm{X}$ \\
\hline Aetheolaena otophora & $\mathrm{X}$ & & \\
\hline Ageratina & & $\mathrm{X}$ & $\mathrm{X}$ \\
\hline Ageratina baccharoides & $\mathrm{X}$ & & \\
\hline Ageratina flaviseta & $\mathrm{X}$ & & \\
\hline Ageratina glyptophlebia & & $\mathrm{X}$ & $\mathrm{X}$ \\
\hline Ageratina gracilis & $\mathrm{X}$ & $\mathrm{X}$ & \\
\hline Ageratina theaefolia & $\mathrm{X}$ & & $\mathrm{X}$ \\
\hline Agrostis & $\mathrm{X}$ & $\mathrm{X}$ & \\
\hline Anredera cordifolia & & $\mathrm{X}$ & $\mathrm{X}$ \\
\hline Anthurium & $\mathrm{X}$ & $\mathrm{X}$ & $\mathrm{X}$ \\
\hline Anthurium oxybelium & $\mathrm{X}$ & $\mathrm{X}$ & $\mathrm{X}$ \\
\hline Aragoa cupressina & $X$ & $\mathrm{X}$ & \\
\hline Arcytophyllum & $\mathrm{X}$ & & \\
\hline Arcytophyllum muticum & $\mathrm{X}$ & $\mathrm{X}$ & \\
\hline Arcytophyllum nitidum & $\mathrm{X}$ & $\mathrm{X}$ & $\mathrm{X}$ \\
\hline Asplenium serra & $\mathrm{X}$ & $\mathrm{X}$ & $\mathrm{X}$ \\
\hline Aulonemia bogotensis & & & $\mathrm{X}$ \\
\hline Baccharis latifolia & & & $\mathrm{X}$ \\
\hline Baccharis rupicola & $\mathrm{X}$ & & \\
\hline Bartsia glandulifera & $\mathrm{X}$ & & \\
\hline Begonia cornuta & & $\mathrm{X}$ & $\mathrm{X}$ \\
\hline Begonia ferruginea & & & $\mathrm{X}$ \\
\hline Bejaria aestuans & $\mathrm{X}$ & $\mathrm{X}$ & $\mathrm{X}$ \\
\hline Bejaria resinosa & $\mathrm{X}$ & & \\
\hline Berberis & $X$ & & \\
\hline Berberis goudotii & $X$ & & \\
\hline Berberis petriruizii & & $\mathrm{X}$ & $\mathrm{X}$ \\
\hline Berberis rigidifolia & $X$ & & \\
\hline Bidens & $X$ & $\mathrm{X}$ & \\
\hline Blechnum columbiense & $X$ & & $X$ \\
\hline Blechnum cordatum & & & $X$ \\
\hline Blechnum loxense & $X$ & $X$ & \\
\hline Bomarea & $X$ & $X$ & $X$ \\
\hline Bomarea setacea & $X$ & $X$ & \\
\hline Brachyotum & $X$ & $X$ & \\
\hline Brunellia & $\mathrm{X}$ & & \\
\hline
\end{tabular}

\begin{tabular}{|c|c|c|c|}
\hline Especie & $\frac{\sqrt{0}}{\mathbb{D}}$ & 营 & 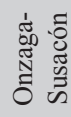 \\
\hline Brunellia colombiana & & $\mathrm{X}$ & \\
\hline Brunellia propinqua & & & $\mathrm{X}$ \\
\hline Bucquetia glutinosa & $\mathrm{X}$ & $\mathrm{X}$ & $\mathrm{X}$ \\
\hline Calamagrostis effusa & $\mathrm{X}$ & $\mathrm{X}$ & $\mathrm{X}$ \\
\hline Campyloneurum amphostenon & $\mathrm{X}$ & & \\
\hline Campyloneurum densifolium & $\mathrm{X}$ & $\mathrm{X}$ & \\
\hline Carex tristachya & $\mathrm{X}$ & $\mathrm{X}$ & \\
\hline Carex tristicha & & $\mathrm{X}$ & \\
\hline Castilleja fissifolia & $\mathrm{X}$ & & $\mathrm{X}$ \\
\hline Castilleja integrifolia & & $\mathrm{X}$ & \\
\hline Castratella piloselloides & $\mathrm{X}$ & $\mathrm{X}$ & \\
\hline Cavendishia & & & $\mathrm{X}$ \\
\hline Cerastium arvense & & $\mathrm{X}$ & \\
\hline Cestrum & & & $\mathrm{X}$ \\
\hline Cestrum buxifolium & $\mathrm{X}$ & & \\
\hline Chusquea & $\mathrm{X}$ & $\mathrm{X}$ & $\mathrm{X}$ \\
\hline Chusquea tessellata & & $\mathrm{X}$ & \\
\hline Clethra fimbriata & $\mathrm{X}$ & $\mathrm{X}$ & \\
\hline Clethra revoluta & $\mathrm{X}$ & & $\mathrm{X}$ \\
\hline Clusia & $\mathrm{X}$ & $\mathrm{X}$ & $\mathrm{X}$ \\
\hline Clusia alata & & & $\mathrm{X}$ \\
\hline Clusia articulata & $\mathrm{X}$ & & $\mathrm{X}$ \\
\hline Clusia elliptica & $\mathrm{X}$ & $\mathrm{X}$ & $\mathrm{X}$ \\
\hline Columnea strigosa & $\mathrm{X}$ & $\mathrm{X}$ & $\mathrm{X}$ \\
\hline Conyza & & $\mathrm{X}$ & \\
\hline Conyza bonariensis & & & $\mathrm{X}$ \\
\hline Cortaderia & $\mathrm{X}$ & $\mathrm{X}$ & $\mathrm{X}$ \\
\hline Culcita coniifolia & $\mathrm{X}$ & $\mathrm{X}$ & \\
\hline Cyathea caracasana & & & $\mathrm{X}$ \\
\hline Cybianthus & & & $\mathrm{X}$ \\
\hline Cybianthus iteoides & $\mathrm{X}$ & & \\
\hline Cybianthus lineatus & $\mathrm{X}$ & & \\
\hline Cynanchum & $\mathrm{X}$ & & \\
\hline Cynanchum microphyllum & $\mathrm{X}$ & & $\mathrm{X}$ \\
\hline Cynanchum tenellum & & & $\mathrm{X}$ \\
\hline Cyrtochilum & & & $\mathrm{X}$ \\
\hline Cyrtochilum revolutum & & $\mathrm{X}$ & \\
\hline Dendrophthora ambigua & $\mathrm{X}$ & & \\
\hline Dendrophthora biserrula & $\mathrm{X}$ & & \\
\hline Dendrophthora clavata & & $\mathrm{X}$ & \\
\hline
\end{tabular}




\begin{tabular}{|c|c|c|c|c|c|c|c|}
\hline Especie & $\frac{\sqrt[0]{0}}{\infty}$ & 䔍 & 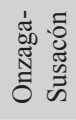 & Especie & $\frac{\sqrt[\cdot]{\tilde{D}}}{\infty}$ & 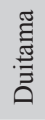 & 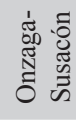 \\
\hline Dendrophthora squamigera & $\mathrm{X}$ & & & Geranium lainzii & $\mathrm{X}$ & & $\mathrm{X}$ \\
\hline Dicksonia sellowiana & & & $\mathrm{X}$ & Geranium multiceps & $\mathrm{X}$ & $\mathrm{X}$ & \\
\hline Diphasiastrum thyoides & $\mathrm{X}$ & $\mathrm{X}$ & $\mathrm{X}$ & Gomphichis & $\mathrm{X}$ & & \\
\hline Diphasium jussiaei & $\mathrm{X}$ & & & Gordonia & $\mathrm{X}$ & & \\
\hline Diplazium & & & $\mathrm{X}$ & Greigia & $\mathrm{X}$ & $\mathrm{X}$ & $\mathrm{X}$ \\
\hline Diplostephium dentatum & $\mathrm{X}$ & & & Greigia danielii & $\mathrm{X}$ & & \\
\hline Diplostephium floribundum & & $\mathrm{X}$ & & Halenia adpressa & & $\mathrm{X}$ & \\
\hline Diplostephium phylicoides & $\mathrm{X}$ & & & Halenia asclepiadea & $\mathrm{X}$ & $\mathrm{X}$ & \\
\hline Diplostephium rosmarinifolius & $\mathrm{X}$ & $\mathrm{X}$ & $\mathrm{X}$ & Halenia gentianoides & $\mathrm{X}$ & & \\
\hline Disterigma & $\mathrm{X}$ & & & Hedyosmum angustifolium & & $\mathrm{X}$ & \\
\hline Disterigma empetrifolium & $\mathrm{X}$ & $\mathrm{X}$ & & Hedyosmum cuatrecazanum & $\mathrm{X}$ & & $\mathrm{X}$ \\
\hline Drimys granadensis & $\mathrm{X}$ & $\mathrm{x}$ & $\mathrm{X}$ & Hesperomeles goudotiana & $\mathrm{X}$ & $\mathrm{X}$ & $\mathrm{X}$ \\
\hline Dryopteris wallichiana & & & $\mathrm{X}$ & Hesperomeles obtusifolia & $\mathrm{x}$ & $\mathrm{X}$ & $\mathrm{X}$ \\
\hline Dysopsis paucidentata & & $\mathrm{X}$ & & Histiopteris incisa & & $\mathrm{X}$ & \\
\hline Elaphoglossum & $\mathrm{X}$ & & & Holcus lanatus & $\mathrm{X}$ & & \\
\hline Elaphoglossum affine & $\mathrm{x}$ & & & Holodiscus argenteus & $\mathrm{x}$ & & \\
\hline Elaphoglossum cuspidatum & & & $\mathrm{X}$ & Huperzia & & & $\mathrm{X}$ \\
\hline Elaphoglossum engelii & & & $\mathrm{x}$ & Hydrocotyle andina & $\mathrm{X}$ & $\mathrm{X}$ & \\
\hline Elaphoglossum eximium & & & $\mathrm{X}$ & Hymenophyllum polyanthos & & $\mathrm{X}$ & \\
\hline Elaphoglossum latifolium & $\mathrm{x}$ & & $\mathrm{x}$ & Hypericum & $\mathrm{X}$ & $\mathrm{X}$ & \\
\hline Elaphoglossum lehmannianum & & $\mathrm{X}$ & & Hypericum brathys & $\mathrm{X}$ & $\mathrm{X}$ & \\
\hline Elaphoglossum lingua & $\mathrm{x}$ & $\mathrm{x}$ & $\mathrm{X}$ & Hypericum cardonae & $\mathrm{x}$ & & \\
\hline Elaphoglossum minutum & & $\mathrm{X}$ & & Hypericum juniperinum & $\mathrm{x}$ & $\mathrm{X}$ & $\mathrm{X}$ \\
\hline Elleanthus & & & $\mathrm{X}$ & Hypericum mexicanum & $\mathrm{x}$ & $\mathrm{X}$ & \\
\hline Elleanthus maculatus & & $\mathrm{X}$ & & Hypericum myricarifolium & $\mathrm{x}$ & & $\mathrm{X}$ \\
\hline Epidendrum & $\mathrm{X}$ & & & Hypericum tetrastichum & $\mathrm{x}$ & & \\
\hline Epidendrum chioneum & $\mathrm{X}$ & & & Hypochaeris radicata & & $\mathrm{X}$ & \\
\hline Eryngium humboldtii & & $\mathrm{X}$ & & Hypochaeris sessiliflora & $\mathrm{X}$ & & \\
\hline Espeletia argentea & $\mathrm{X}$ & & $\mathrm{X}$ & Hypochaeris setosa & $\mathrm{X}$ & & \\
\hline Espeletia boyacensis & $\mathrm{X}$ & & $\mathrm{x}$ & Hypolepis & $\mathrm{x}$ & & \\
\hline Espeletia brachyaxiantha & $\mathrm{X}$ & & & Ilex & & $\mathrm{X}$ & \\
\hline Espeletia congestiflora & & $\mathrm{X}$ & & Ilex kunthiana & $\mathrm{X}$ & $\mathrm{X}$ & \\
\hline Espeletia murilloi & & $\mathrm{X}$ & & Ilex laurina & $\mathrm{X}$ & & $\mathrm{X}$ \\
\hline Eugenia & $\mathrm{X}$ & & & Ilex nervosa & & $\mathrm{X}$ & \\
\hline Festuca & & $\mathrm{X}$ & & Ilex obtusata & & & $\mathrm{X}$ \\
\hline Gaiadendron punctatum & $\mathrm{X}$ & $\mathrm{X}$ & $\mathrm{X}$ & Jamesonia & & $\mathrm{X}$ & \\
\hline Galium & $\mathrm{x}$ & & & Jamesonia imbricata & & & $\mathrm{X}$ \\
\hline Galium corymbosum & & & $\mathrm{X}$ & Jungia & & $\mathrm{X}$ & \\
\hline Galium hypocarpium & $\mathrm{X}$ & $\mathrm{X}$ & & Lachemilla fulvescens & & & $\mathrm{X}$ \\
\hline Gaultheria anastomosans & $\mathrm{x}$ & $\mathrm{x}$ & $\mathrm{X}$ & Lachemilla nivalis & $\mathrm{X}$ & $\mathrm{X}$ & \\
\hline Gaultheria erecta & & & $\mathrm{X}$ & Lepanthes & & & $\mathrm{X}$ \\
\hline Gaultheria lanigera & & $\mathrm{X}$ & & Lepechinia conferta & $\mathrm{X}$ & & \\
\hline Gaultheria rigida & $\mathrm{X}$ & $\mathrm{X}$ & & Luzula gigantea & $\mathrm{X}$ & & \\
\hline Gaultheria strigosa & & & $\mathrm{X}$ & Lycopodium clavatum & $\mathrm{x}$ & $\mathrm{X}$ & $\mathrm{X}$ \\
\hline Gaylussacia buxifolia & & $\mathrm{X}$ & $\mathrm{X}$ & Macleania rupestris & $\mathrm{X}$ & $\mathrm{X}$ & $\mathrm{X}$ \\
\hline Gentianella corymbosa & & $\mathrm{X}$ & & Masdevallia & & $\mathrm{X}$ & \\
\hline
\end{tabular}




\begin{tabular}{|c|c|c|c|c|c|c|c|}
\hline Especie & $\frac{\sqrt[0]{0}}{\infty}$ & 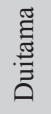 & 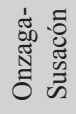 & Especie & $\frac{\sqrt[0]{0}}{\tilde{D}}$ & 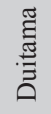 & 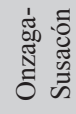 \\
\hline Melpomene assurgens & $\mathrm{X}$ & & & Palicourea aschersonianoides & $\mathrm{X}$ & & \\
\hline Melpomene moniliformis & & $\mathrm{X}$ & & Palicourea demissa & & & $\mathrm{X}$ \\
\hline Meriania brachycera & $\mathrm{X}$ & & $\mathrm{X}$ & Paragynoxys neodendroides & & $\mathrm{x}$ & $\mathrm{X}$ \\
\hline Miconia & & $\mathrm{X}$ & & Paramiflos glandulosus & & $\mathrm{X}$ & \\
\hline Miconia alborosea & $\mathrm{X}$ & $\mathrm{X}$ & & Paspalum & $\mathrm{X}$ & & \\
\hline Miconia biglandulosa & & & $\mathrm{X}$ & Passiflora & & & $\mathrm{X}$ \\
\hline Miconia buxifolia & $\mathrm{X}$ & $\mathrm{X}$ & & Pentacalia & & $\mathrm{X}$ & \\
\hline Miconia cataractae & $\mathrm{X}$ & & $\mathrm{X}$ & Pentacalia abietina & $\mathrm{X}$ & $\mathrm{X}$ & \\
\hline Miconia cladonia & $\mathrm{X}$ & & & Pentacalia corymbosa & $\mathrm{X}$ & & $\mathrm{X}$ \\
\hline Miconia cundinamarcensis & $\mathrm{X}$ & & & Pentacalia ledifolia & & $\mathrm{X}$ & $\mathrm{X}$ \\
\hline Miconia elaeoides & & $\mathrm{X}$ & & Pentacalia pulchella & $\mathrm{X}$ & $\mathrm{X}$ & \\
\hline Miconia jahnii & & $\mathrm{x}$ & & Pentacalia vaccinioides & $\mathrm{X}$ & $\mathrm{X}$ & \\
\hline Miconia latifolia & $\mathrm{X}$ & & & Peperomia & $\mathrm{x}$ & & \\
\hline Miconia rigens & & & $\mathrm{X}$ & Peperomia acuminata & & & $\mathrm{X}$ \\
\hline Miconia salicifolia & & $\mathrm{X}$ & & Peperomia ambiguifolia & $\mathrm{X}$ & $\mathrm{X}$ & $\mathrm{X}$ \\
\hline Miconia squamulosa & & & $\mathrm{X}$ & Peperomia galioides & $\mathrm{X}$ & & \\
\hline Miconia summa & & $\mathrm{X}$ & & Peperomia leucostachya & $\mathrm{X}$ & & \\
\hline Monnina aestuans & $\mathrm{X}$ & & & Pernettya prostrata & & $\mathrm{X}$ & $\mathrm{X}$ \\
\hline Monnina densa & & $\mathrm{X}$ & & Phoradendron & $\mathrm{X}$ & & \\
\hline Monnina salicifolia & $\mathrm{X}$ & $\mathrm{x}$ & $\mathrm{X}$ & Piper artanthe & & & $\mathrm{x}$ \\
\hline Monochaetum myrtoideum & $\mathrm{X}$ & & & Piptocarpha poeppigiana & & & $\mathrm{X}$ \\
\hline Muehlenbeckia tamnifolia & & & $\mathrm{X}$ & Plagiogyria semicordata & $\mathrm{X}$ & & \\
\hline Munnozia & $\mathrm{X}$ & & & Pleopeltis murora & & & $\mathrm{X}$ \\
\hline Munnozia senecionidis & & $\mathrm{X}$ & $\mathrm{X}$ & Pleurothallis & $\mathrm{X}$ & $\mathrm{X}$ & \\
\hline Myrsine & & $\mathrm{x}$ & & Pleurothallis bivalvis & & & $\mathrm{x}$ \\
\hline Myrsine dependens & $\mathrm{X}$ & $\mathrm{x}$ & $\mathrm{X}$ & Pleurothallis lindenii & & & $\mathrm{X}$ \\
\hline Myrsine guianensis & $\mathrm{X}$ & $\mathrm{x}$ & $\mathrm{X}$ & Polystichum orbiculatum & $\mathrm{X}$ & & \\
\hline Myrteola nummularia & $\mathrm{X}$ & & & Psammisia graebneriana & $\mathrm{X}$ & $\mathrm{X}$ & $\mathrm{X}$ \\
\hline Nassella & & & $\mathrm{x}$ & Psychotria & & & $\mathrm{X}$ \\
\hline Nectandra & & & $\mathrm{x}$ & Puya nitida & $\mathrm{X}$ & & \\
\hline Nertera granadensis & $\mathrm{X}$ & X & $\mathrm{X}$ & Puya santosii & & $\mathrm{X}$ & $\mathrm{X}$ \\
\hline Niphogeton glaucescens & $\mathrm{X}$ & & & Quercus humboldtii & $\mathrm{X}$ & $\mathrm{X}$ & $\mathrm{X}$ \\
\hline Ocotea calophylla & $\mathrm{X}$ & $\mathrm{X}$ & $\mathrm{X}$ & Racinaea tetrantha & & & $\mathrm{X}$ \\
\hline Ocotea guianensis & $\mathrm{X}$ & & $\mathrm{X}$ & Rhamnus & $X$ & & $\mathrm{X}$ \\
\hline Oncidium ornithorhynchum & & $\mathrm{x}$ & & Rhamnus goudotiana & & $\mathrm{X}$ & $\mathrm{X}$ \\
\hline Oreobolus cleefii & $\mathrm{X}$ & $\mathrm{x}$ & & Rhamnus sphaerosperma & & & $\mathrm{X}$ \\
\hline Oreopanax bogotensis & & $\mathrm{x}$ & & Rhynchospora & & $\mathrm{X}$ & \\
\hline Ortachne erectifolia & $\mathrm{X}$ & & & Rhynchospora ruiziana & $\mathrm{X}$ & $\mathrm{X}$ & $\mathrm{X}$ \\
\hline Orthrosanthus & $\mathrm{X}$ & & & Ribes & $\mathrm{x}$ & & \\
\hline Orthrosanthus chimboracensis & $\mathrm{x}$ & $\mathrm{X}$ & $\mathrm{X}$ & Ribes andicola & $\mathrm{x}$ & & \\
\hline Oxalis & & & $\mathrm{X}$ & Ribes bogotanum & & $\mathrm{X}$ & \\
\hline Oxalis medicaginea & & $\mathrm{X}$ & & Rubus acanthophyllos & $\mathrm{x}$ & $\mathrm{X}$ & \\
\hline Paepalanthus andicola & $\mathrm{X}$ & $\mathrm{x}$ & & Rubus bogotensis & $\mathrm{X}$ & & \\
\hline Paepalanthus columbiensis & $\mathrm{x}$ & & $\mathrm{X}$ & Rubus macrocarpus & & $\mathrm{X}$ & $\mathrm{X}$ \\
\hline Paepalanthus karstenii & $\mathrm{X}$ & & & Rubus robustus & & & $\mathrm{X}$ \\
\hline Palicourea apicata & & $\mathrm{x}$ & $\mathrm{X}$ & Salpichroa tristis & & & $\mathrm{X}$ \\
\hline
\end{tabular}




\begin{tabular}{|c|c|c|c|}
\hline Especie & $\frac{\text { లే }}{\stackrel{0}{D}}$ & 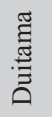 & 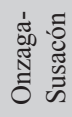 \\
\hline Schefflera & & & $\mathrm{X}$ \\
\hline Schefflera bogotensis & $\mathrm{X}$ & $\mathrm{X}$ & \\
\hline Schefflera trianae & $\mathrm{X}$ & $\mathrm{X}$ & \\
\hline Scrobicaria ilicifolia & $\mathrm{X}$ & $\mathrm{X}$ & $\mathrm{X}$ \\
\hline Serpocaulon & & $\mathrm{X}$ & \\
\hline Serpocaulon lasiopus & & & $\mathrm{X}$ \\
\hline Siphocampylus & $\mathrm{X}$ & & \\
\hline Smilax & & & $X$ \\
\hline Smilax floribunda & & $\mathrm{X}$ & \\
\hline Solanum & & & $X$ \\
\hline Solanum nigrum & & & $X$ \\
\hline Solanum oblongifolium & & & $\mathrm{X}$ \\
\hline Solanum pseudocapsicum & & & $X$ \\
\hline Sphyrospermum & $\mathrm{X}$ & $\mathrm{X}$ & \\
\hline Sporobolus & & & $\mathrm{X}$ \\
\hline Stellaria & & & $\mathrm{X}$ \\
\hline Stellis & $\mathrm{X}$ & $\mathrm{X}$ & $\mathrm{X}$ \\
\hline Styrax davillifolius & & & $\mathrm{X}$ \\
\hline Symplocos cundinamarcensis & $\mathrm{X}$ & $\mathrm{X}$ & \\
\hline
\end{tabular}

\begin{tabular}{|c|c|c|c|}
\hline Especie & $\frac{\sqrt[0]{0}}{\stackrel{0}{0}}$ & 节 & 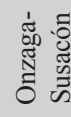 \\
\hline Symplocos theiformis & $\mathrm{X}$ & $\mathrm{X}$ & $\mathrm{X}$ \\
\hline Ternstroemia & $\mathrm{X}$ & $\mathrm{X}$ & \\
\hline Ternstroemia macrocarpa & & & $\mathrm{X}$ \\
\hline Ternstroemia meridionalis & $\mathrm{X}$ & & $\mathrm{X}$ \\
\hline Thibaudia & & & $\mathrm{X}$ \\
\hline Tibouchina grossa & & $\mathrm{X}$ & \\
\hline Ugni myricoides & $\mathrm{X}$ & $\mathrm{X}$ & \\
\hline Uncinia & $\mathrm{X}$ & & \\
\hline Vaccinium & & $\mathrm{X}$ & \\
\hline Vaccinium floribundum & $\mathrm{X}$ & $\mathrm{X}$ & $\mathrm{X}$ \\
\hline Vaccinium meridionale & & $\mathrm{X}$ & $\mathrm{X}$ \\
\hline Valeriana clematitis & $\mathrm{X}$ & & \\
\hline Valeriana vetasana & $\mathrm{X}$ & & \\
\hline Vallea stipularis & $\mathrm{X}$ & $\mathrm{X}$ & $\mathrm{X}$ \\
\hline Viburnum tinoides & & & $\mathrm{X}$ \\
\hline Viburnum triphyllum & & $\mathrm{X}$ & $\mathrm{X}$ \\
\hline Weinmannia fagaroides & $\mathrm{X}$ & $\mathrm{X}$ & $\mathrm{X}$ \\
\hline Weinmannia tomentosa & $\mathrm{X}$ & $\mathrm{X}$ & $\mathrm{X}$ \\
\hline
\end{tabular}

